\title{
Erratum to: Reaction of small heat-shock proteins to different kinds of cellular stress in cultured rat hippocampal neurons
}

\author{
Britta Bartelt-Kirbach • Nikola Golenhofen
}

Published online: 6 November 2013

(C) Cell Stress Society International 2013

\section{Erratum to: Cell Stress and Chaperones \\ DOI 10.1007/s12192-013-0452-9}

Due to an unfortunate mistake during the production process, the full captions of Figures 1 and 2 were erroneously placed as regular text at the end of the article instead of as proper captions. In order to rectify this error, the Figures and their captions as well as the Discussion section are published below and on the following pages and should be treated as definitive by the reader.

\section{Discussion}

sHsps in neurons after different kinds of stress

Small heat-shock proteins play a major role in the development of cellular stress tolerance in the brain. This phenomenon, which has been described after many different types of stress, is also known for cross-tolerance, meaning that after preconditioned with one kind of stress the cell tolerates also others (Kirino 2002; Plamondon et al. 1999). Studies on sHsps in neurodegenerative diseases (Muchowski and Wacker 2005) as well as studies on hereditary distal motor neuropathies caused by mutations in some sHsp genes (Mymrikov et al. 2011) may suggest a neuroprotective function of sHsps. However, so far, most studies investigating the role of

The online version of the original article can be found under doi:10.1007/ s12192-013-0452-9.

B. Bartelt-Kirbach $\cdot$ N. Golenhofen $(\bowtie)$

Institute of Anatomy and Cell Biology, University of Ulm,

Albert-Einstein-Allee 11, 89081 Ulm, Germany

e-mail: nikola.golenhofen@uni-ulm.de
sHsps in neuroprotection focused on one selected sHsp instead of a comparative analysis of all sHsps. This is the first study investigating systematically the response of all expressed sHsps to three different kinds of stress in hippocampal neurons, i.e., sodium arsenite and oxidative and hyperosmotic stress. Sodium arsenite stress was chosen to mimic an exposure to a neurotoxic chemical substance. Oxidative stress was investigated as it is well known to be involved in neurodegenerative diseases (Ebenezer et al. 2010) and also plays a part in ischemia (Levraut et al. 2003). Finally, hyperosmotic stress was chosen because neurons in the brain have to cope with osmotic imbalances in blood plasma (Loyher et al. 2004).

Previously, we found that rat hippocampal neurons express HspB1, HspB5, HspB6, HspB8, and HspB11, but sublethal heat shock led only to the upregulation of HspB1 and HspB5 proteins (Kirbach and Golenhofen 2011). In the present study, we could demonstrate that not only HspB1 and $\mathrm{HspB} 5$ but also HspB6 and HspB8 protein amount was upregulated in cultured hippocampal neurons in response to all three abovementioned stress conditions. It is interesting that such diverse conditions led to a relative consistent reaction. In the literature, HspB1 is the one sHsp studied most extensively so far. It has been found upregulated in neurons after ischemia (Kalesnykas et al. 2008; Kato et al. 1994; Lu et al. 2002), heat shock (Bechtold and Brown 2000; Krueger-Naug et al. 2000; Satoh and Kim 1995; Schwarz et al. 2010), hypoxia (David et al. 2006), oxidative stress (Schwarz et al. 2010), kainic acid induced seizures (Kato et al. 1999), exposure to electromagnetic fields (Yang et al. 2012) or toxic substances (Clark et al. 2011), and during axonal regeneration (Hebb et al. 2006). HspB5 has previously been found upregulated in neurons after ischemia (Minami et al. 2003; Piao et al. 2005), olivary hypertrophy (Fukushima et al. 2006), heat shock, and oxidative stress (Schwarz et al. 2010). Interestingly, HspB5 is recruited to 

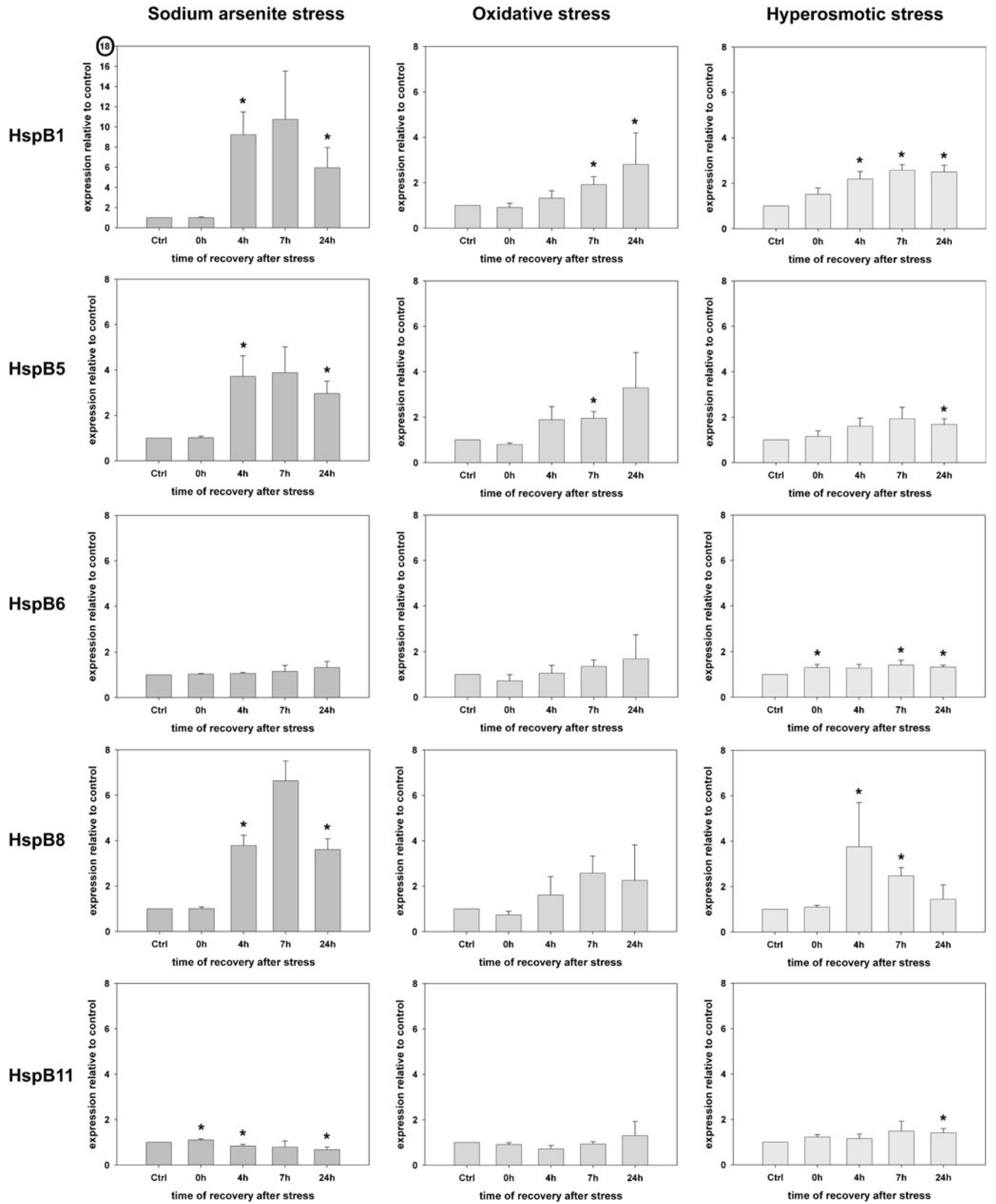
4 Figure 1 Changes of mRNA amount in cultured rat hippocampal neurons after different kinds of stress. mRNA amount of HspB1, HspB5, HspB6, HspB8, and HspB11 relative to control after sodium arsenite, oxidative or hyperosmotic stress (first, second, and third columns, respectively). Data from at least three independent experiments are presented as mean $\pm \mathrm{SEM}$. The non-parametric MannWhitney $U$ test was used for statistical analysis, ${ }^{*} p<0.05$. Rat hippocampal neurons prepared from embryonal hippocampi (E18-E19) of Sprague-Dawley rats as described previously (Kirbach and Golenhofen 2011) were seeded at a density of $30,000 \mathrm{cells} / \mathrm{cm}^{2}$ in Petri dishes and grown in Neurobasal ${ }^{\mathrm{TM}}$ medium supplemented with B27 ${ }^{\circledR}$ (Life Technologies GmbH, Darmstadt, Germany) for 14 days. At DIV14, cells were exposed to sodium arsenite stress $(100 \mu \mathrm{M}$ sodium arsenite, 30 min), oxidative stress $(0.5 \mu \mathrm{M}$ hydrogen peroxide, $30 \mathrm{~min})$, or hyperosmotic stress $(200 \mu \mathrm{M}$ sorbitol, $2 \mathrm{~h})$. After stress, cells received conditioned medium from cultures without stress of the same preparation. After the indicated time points of recovery cells were lysed and total RNA isolated, DNase treated and reverse-transcribed as described (Kirbach and Golenhofen 2011). Real-time PCR was performed as described previously (Kirbach and Golenhofen 2011) with $2 \mu 1$ 1:10 diluted cDNA and the QuantiTect SYBR Green PCR Kit (Qiagen, Hilden, Germany). Primers used for amplification are listed in Table 1. Original fluorescence $F_{0}$ was calculated according to Rutledge and Cote (2003). The geometric mean of both reference genes (cyclophilin A and Rpl13A) was used for normalization. The resulting values were then calculated in relation to the control sample of the respective stress experiment, which was set to 1 .

dendrites and synapses in response to phosphorylation (Schmidt et al. 2012). Little is known about the regulation of HspB6 and HspB8 in neurons. HspB6 was described to be upregulated after ischemia (Niwa et al. 2009) and hypoxia (David et al. 2006) while for HspB8 an increase was found after proteasome inhibition (Yew et al. 2005). Taking all these data on single sHsps together with our findings, it seems obvious that neurons upregulate more than one sHsp under stress conditions and that HspB1, HspB5, HspB6, and HspB8 seem to act together in protecting them from damage. Focusing on only one sHsp therefore means missing a major part of the sHsp response in neurons.

\section{Posttranscriptional regulation of sHsps}

Concerning the time course of the stress response, we found the peak of protein amount around $24 \mathrm{~h}$ of recovery after stress for most sHsps followed by a subsequent decrease. This seemed largely independent of the kind of stress applied. Interestingly, we observed the increase on mRNA level earlier (mostly already after $4 \mathrm{~h}$ of recovery) as at the protein level (starting only after $7 \mathrm{~h}$ of recovery). This discrepancy was observed by us also after heat shock (Kirbach and Golenhofen 2011). It is well known that exposure to stress leads to a translational arrest followed by a selective translation of stress related transcripts (DeGracia et al. 2008; Fahling 2009; Lindquist 1986). Mizzen andWelch (1988) observed that this translational arrest lasted up to $5 \mathrm{~h}$ after heat shock, a time frame
Figure 2 Changes of protein amount in rat hippocampal neurons after different kinds of stress. Protein amount of HspB1, HspB5, HspB6, HspB8, and HspB11 after (A) sodium arsenite stress, (B) oxidative stress, and (C) hyperosmotic stress. Representative immunoblots from at least three independent experiments are shown. Equal amount of protein loading was controlled by PonceauS staining which is shown exemplarily for the HspB1 blot after arsenite stress. Cells were cultured and stressed as described in Fig. 1. After the indicated time points of recovery, cells were lysed with Laemmli buffer and protein concentration determined by amido black staining (Dieckmann-Schuppert and Schnittler 1997; Heinzel et al. 1965). Immunoblots were carried out as described (Kirbach and Golenhofen 2011) with equal amounts of protein (20 $\mu \mathrm{g}$ per lane) after separation with SDS-PAGE on $15 \%$ acrylamide gels. Membranes were stained for 2 min with $0.5 \%$ PonceauS, $3 \%$ trichloroacetic acid to control equal protein loading and transfer. For the detection of sHsps, the following antibodies were used: rabbit anti-HspB1 (1:1000, Stressgen, SPA-801), rabbit anti-HspB5 (1:500, Stressgen, SPA223), rabbit anti-HspB5 (1:5000, Lifespan, LSC105475), goat antiHspB6 (1:1000, R\&D Systems, AF4200), rabbit anti-HspB8 (1:750, Cell Signaling, 3059), rabbit anti-HspB11 (1:1000, kind gift of Prof. Dr. Bellyei, Dept. of Oncotherapy, University of Pécs, Hungary). All antibodies were incubated in blocking solution containing $5 \%$ milk except for the anti-HspB5 antibody from Lifespan which was incubated with $5 \%$ bovine serum albumin or $1 \%$ milk. Secondary antibodies were horesradish-peroxidase labelled goat anti-rabbit IgG (1:5000, Jackson Immuno Research Laboratories) or rabbit anti-goat IgG (1:2000, Dako). Washing steps were performed using TBS with $0.05 \%$ Tween 20 . Detection of bound antibodies was carried out with the enhanced chemoluminescence system (Pierce ECL Western Blotting Substrate, Thermo Fisher Scientific Inc.) on X-ray films (Hyperfilm ECL, GE Healthcare).

fitting to our observation. In two cases, a prolonged increase of protein amount up to $72 \mathrm{~h}$ of recovery was measured: for $\mathrm{HspB} 1$ after sodium arsenite and for $\mathrm{HspB} 8$ after hyperosmotic stress. Interestingly, the mRNA amount in both cases already started to decrease after $24 \mathrm{~h}$ of recovery. This hints at a posttranscriptional regulation mechanism involved in the stress response.It was recently shown in heart muscle that $\mathrm{HspB} 6$ is regulated by the microRNA miR-320 (Ren et al. 2009). MicroRNAs are small non-coding RNAs which are incorporated in the RNA-induced silencing complex, bind to the 3'UTR of target mRNAs and inhibit their translation (Boyd 2008). This microRNA could therefore play a role in the regulation of HspB6 in neurons. Whether microRNAs also play a role in the regulation of HspB1, HspB5, and HspB8 remains to be investigated.

\section{HspB11—a special case}

The fifth sHsp expressed in rat hippocampal neurons, HspB11, showed an inconsistent regulation in our experiments. Mostly, no change in protein or mRNA amount was observed after stress while in some instances up- or downregulation was detectable. This is in concordance with our previous finding after heat shock where no 
A Sodium arsenite stress

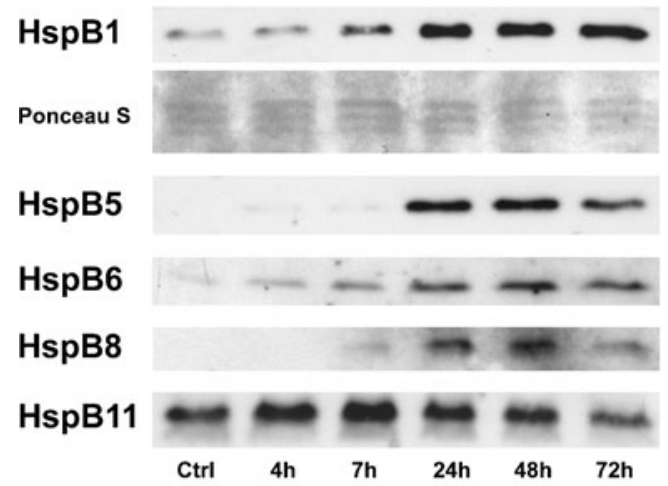

B Oxidative stress

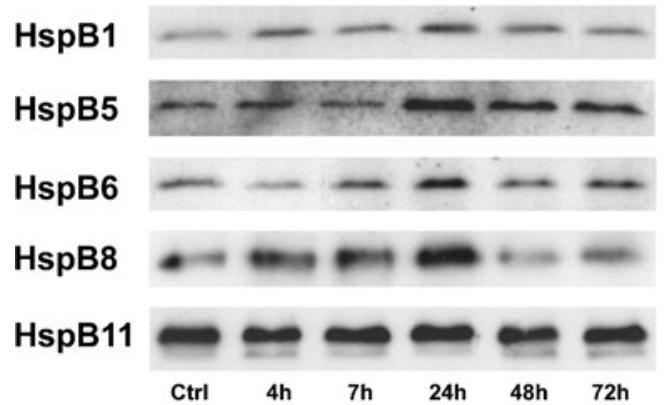

C Hyperosmotic stress

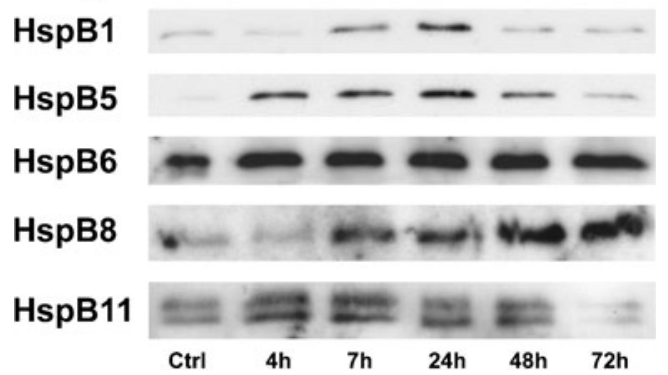

upregulation was observed (Kirbach and Golenhofen 2011).

HspB11 was first described by Bellyei et al. (2007) as a novel sHsp (Hsp16.2). They reported a homology to the alpha-crystallin domain and also a cytoprotective effect by stabilization of the mitochondrial membrane. However, it is still under debate if this protein belongs to the sHsp family at all, as the described homology with the alphacrystallin domain was not confirmed by a more detailed approach (Kappe et al. 2010). Our data also suggest that HspB11 does not act primarily as a chaperone after stress but may have a constitutive function. It has been implicated to be a part of the intraflagellate transport complex B of primary cilia (Follit et al. 2009) where it seems to be required for Hedgehog signaling (Keady et al. 2012). It would therefore be of interest if it aids in cilia signaling in neurons.

In conclusion, we could show that four small heat-shock proteins (HspB1, HspB5, HspB6, HspB8) are induced in rat hippocampal neurons after sodium arsenite and oxidative and hyperosmotic stress whereas only HspB1 and HspB5 were upregulated after heat shock. We found variations in the time course of the responses which might be due to posttranscriptional regulation. These four sHsps seem to act together to protect neurons from adverse conditions. It is not clear if this protection is mediated by their chaperone activity alone or by their individual specific functions. The controversial sHsp HspB11 does not seem to play a role in the stress response under these conditions.

Acknowledgments We would like to thank BiancaMekle, Diana Reinhardt, and Silke Zschemisch for their expert technical assistance. 